\title{
Progressive thrombocytopenia after cardiac surgery in a 67-year-old man
}

\author{
Ilana Kopolovic MD, Theodore E. Warkentin MD
}

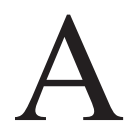
67-year-old man who had undergone uncomplicated bioprosthetic replacement of the aortic valve and coronary artery bypass grafting developed transient postoperative thrombocytopenia, followed by a second decline in platelet count. He was clinically stable and did not have new symptoms or postoperative complications. There was no evidence of sepsis, thrombosis or hemorrhage. The patient had received unfractionated heparin (70 $000 \mathrm{IU}$ in total) with protamine reversal $(600 \mathrm{mg})$, one unit of pooled platelets and one unit of packed red blood cells during the operation. Enoxaparin $(30 \mathrm{mg}$ twice daily) had been given postoperatively for antithrombotic prophylaxis.

The patient's platelet count fell to $43 \times 10^{\circ} / \mathrm{L}$ by the second postoperative day and then gradually rose to $85 \times 10^{9} / \mathrm{L}$ by the fifth day. His platelet count began to decline for a second time on day 6 after surgery and reached $47 \times 10^{9} / \mathrm{L}$ on day 7 , which prompted a consultation with the hematology service (Box 1). A complete blood count showed that the patient had mild, stable, postoperative anemia (hemoglobin level $82 \mathrm{~g} / \mathrm{L}$ and mean corpuscular volume $95 \mathrm{fL}$ ) and a normal leukocyte count $\left(6.2 \times 10^{9} / \mathrm{L}\right)$ with a normal differential. A peripheral blood film confirmed thrombocytopenia, with no morphologic abnormalities in the erythrocytes or leukocytes. Prothrombin and activated partial thromboplastin times were within normal limits. The patient's lactate dehydrogenase level was 207 (normal 125-220) U/L.

\section{What is the most likely diagnosis at the seventh postoperative day?}
a. Dilutional thrombocytopenia
b. Heparin-induced thrombocytopenia
c. Post-transfusion purpura
d. Thrombotic thrombocytopenic purpura

A tentative diagnosis of heparin-induced thrombocytopenia, an immune-mediated thrombocytopenia resulting in platelet activation and hypercoagulability, was made (b). Dilutional thrombocytopenia could explain the initial decline in platelet count, which reached its nadir on the second postoperative day, and the subsequent recovery of the platelet count to $85 \times 10^{9} / \mathrm{L}$. The nadir of postoperative thrombocytopenia can occur anytime between days 1 and 4 after surgery (most commonly occuring on day 2). Once the platelet count begins to rise, the ultimate peak count occurs on about day 14, with a gradual restoration to preoperative levels over the following one to two weeks. ${ }^{1}$ In contrast, new-onset thrombocytopenia on or after postoperative day 5 , which was observed in our patient, is not consistent with a diagnosis of dilutional thrombocytopenia.

Post-transfusion purpura is a rare disorder that occurs almost exclusively in women with a remote history of pregnancy or transfusion; less than 5\% of cases are seen in men who have had previous blood transfusion. ${ }^{2}$ Post-transfusion purpura presents with severe thrombocytopenia (platelet count $<10 \times$ $10^{\circ} / \mathrm{L}$ ) and mucocutaneous bleeding that occurs 510 days after transfusion with a platelet-containing product (usually packed red blood cells). ${ }^{2}$

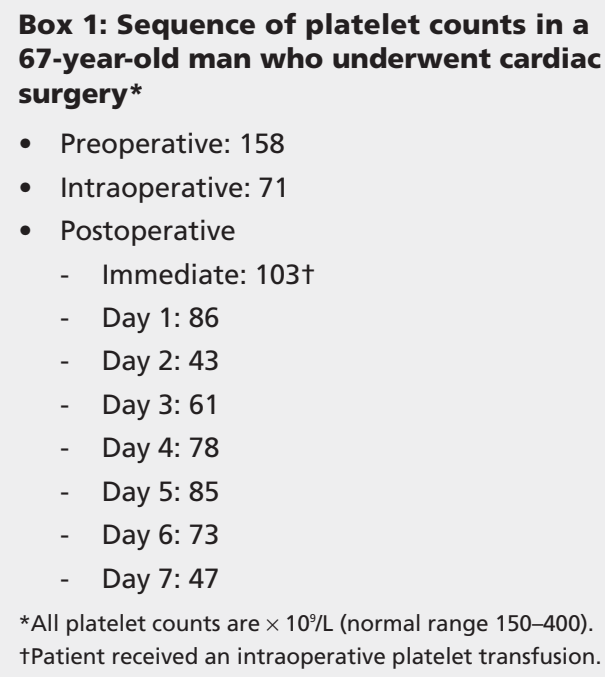

*All platelet counts are $\times 10^{\circ} / \mathrm{L}$ (normal range 150-400). tPatient received an intraoperative platelet transfusion.

Competing interests: Theodore Warkentin has received a grant from the Heart and Stroke Foundation of Ontario. He has received lecture honoraria from Canyon Pharmaceuticals, Instrumentation Laboratory and Pfizer Canada; and he has received consulting fees from ParinGenix and W.L.

Gore, as well as fees for medical-legal testimony. $\mathrm{He}$ has received royalties from Taylor \& Francis. No competing interests were declared by Ilana Kopolovic.

This article has been peer reviewed.

Correspondence to: Ilana Kopolovic, kopolovi@ualberta.ca

CMAJ 2014. DOI:10.1503 /cmaj.131449 
Thrombotic thrombocytopenic purpura can occur postoperatively, in particular following cardiac surgery, ${ }^{3}$ but it is rare. The absence of schistocytes on the blood film and a normal lactate dehydrogenase level ruled out this diagnosis in our patient.

A presumptive diagnosis of heparin-induced thrombocytopenia was made, and a therapeutic dose of fondaparinux $(7.5 \mathrm{mg} / \mathrm{d})$ was started based on expert opinion. ${ }^{4} \mathrm{~A}$ polyspecific enzyme-linked immunosorbent assay (ELISA) for antiplatelet factor 4/heparin antibodies was strongly positive (optical density 2.5 [normal $<0.40]$ ). Bilateral Doppler and compression ultrasound scans of the legs were obtained to rule out subclinical thrombosis, ${ }^{5}$ because its presence would influence the required duration of anticoagulation. The ultrasound images showed no evidence of deep vein thrombosis, and there were no clinical signs or symptoms of other venous or arterial thromboembolism.

Despite the change to treatment with fondaparinux, the patient's platelet count continued to decline over the next four days, from $47 \times 10^{\circ} / \mathrm{L}$ on postoperative day 7 to $13 \times 10^{9} / \mathrm{L}$ on day 11 . All other parameters of the complete blood count and peripheral blood film were unchanged. Prothrombin and activated thromboplastin times remained within normal limits, and the fibrinogen level was elevated (4.5 [normal 1.53.5] g/L). The hematology service was asked to reevaluate therapy.

\section{What is the most appropriate intervention for this patient?}

a. Stop treatment with fondaparinux and start high-dose intravenous immunoglobulin treatment

b. Stop treatment with fondaparinux and start treatment with a direct thrombin inhibitor

c. Continue treatment with fondaparinux

d. Stop treatment with fondaparinux without starting treatment with a different anticoagulant

Fondaparinux treatment was continued (c) for a presumptive diagnosis of heparin-induced thrombocytopenia, based on the presence of antibodies characteristic of the "delayed-onset" type. High-dose intravenous immunoglobulin treatment would be an appropriate treatment for posttransfusion purpura. A direct thrombin inhibitor, such as argatroban or bivalirudin, would be an appropriate treatment for heparin-induced thrombocytopenia, in keeping with recent evidencebased guidelines, ${ }^{6}$ however, titration of the dose of these agents using the laboratory coagulation parameters can be confounded by concomitant disseminated intravascular coagulation, ${ }^{7}$ a complication occurring in up to $25 \%$ of patients with delayed-onset heparin-induced thrombocytopenia. ${ }^{8,9}$ A case series of patients with welldocumented heparin-induced thrombocytopenia reported that fondaparinux was a safe and effective treatment for this condition..$^{10}$ The occurrence or perpetuation of heparin-induced thrombocytopenia in patients taking fondaparinux is rare. ${ }^{11}$ Most importantly, the usual progression of the delayed-onset variant of heparin-induced thrombocytopenia is characterized by progressive thrombocytopenia that reaches its nadir between days 10 and 17 after surgery. 8,9

Given that our patient was otherwise doing well, without clinical evidence of thrombosis or overt disseminated intravascular coagulation, the decision was made to continue treatment with fondaparinux. Discontinuation of fondaparinux without starting treatment with a different anticoagulant would put the patient at risk of venous or arterial thromboembolism, which occurs in over $50 \%$ of patients with heparin-induced thrombocytopenia if adequate anticoagulation is not provided. ${ }^{12}$ Current guidelines and an expert review recommend a minimum of four weeks of therapeutic treatment with a non-heparin anticoagulant in patients who have heparin-induced thrombocytopenia without thrombosis. ${ }^{5,6}$

\section{Which result of a serotonin release assay would confirm the diagnosis?}

a. Serotonin release at a dose of $0.1 \mathrm{IU} / \mathrm{mL}$ heparin, but not at a dose of 0 or $100 \mathrm{IU} / \mathrm{mL}$ heparin

b. Serotonin release at doses of 0 and $0.1 \mathrm{IU} / \mathrm{mL}$ heparin, but not at a dose of $100 \mathrm{IU} / \mathrm{mL}$ heparin

c. No serotonin release at any concentration of heparin

d. Serotonin release only with doses of fondaparinux

The serotonin release assay is a diagnostic test for heparin-induced thrombocytopenia that measures the ability of the antiplatelet factor 4 /heparin immunoglobulin $\mathrm{G}$ (IgG) antibodies to activate platelets and induce serotonin release in a sample of serum. In our patient, the results of the assay confirmed strong reactivity (b) both in the presence of heparin at a therapeutic dose (day 11 serum sample: peak $92 \%$ serotonin release at $0.1 \mathrm{IU} / \mathrm{mL}$ unfractionated heparin) and in the absence of heparin (peak $81 \%$ serotonin release at $0 \mathrm{IU} / \mathrm{mL}$ unfractionated heparin) 
(Appendix 1, available at www.cmaj.ca/lookup /suppl/doi:10.1503/cmaj.131449/-/DC1). Platelet activation was inhibited at a high concentration of heparin $(0 \%$ serotonin release at a dose of $100 \mathrm{IU} / \mathrm{mL}$ unfractionated heparin). The strong serum-induced activation of platelets observed in the absence of heparin (buffer control) is an important serologic feature of delayed-onset heparin-induced thrombocytopenia. ${ }^{9}$

Serotonin release at a dose of $0.1 \mathrm{IU} / \mathrm{mL}$ heparin, but not a dose of 0 or $100 \mathrm{IU} / \mathrm{mL}$ heparin, would be characteristic of most patients with heparin-induced thrombocytopenia, in whom strong reactivity in the serotonin release assay is observed only when the patient is receiving therapeutic concentrations of heparin. No serotonin release at any concentration of heparin argues against a diagnosis of heparin-induced thrombocytopenia.

Serotonin release only with doses of fondaparinux would implicate fondaparinux as a causal agent of the thrombocytopenia and would suggest that this agent was provoking platelet activation and an associated prothrombotic state. There was no increase in reactivity over the buffer control at any dose of fondaparinux tested; this included tests of patient serum diluted 1:16 and 1:64, in which less than $5 \%$ reactivity was observed at a dose of $0 \mathrm{IU} / \mathrm{mL}$ heparin and at all concentrations of fondaparinux, but strong heparin-dependent activation of platelets ( $>80 \%$ serotonin release) remained (Appendix 1). Because the addition of fondaparinux did not increase the degree of serotonin release compared with that observed with the buffer control, fondaparinux cross-reactivity was ruled out.

The patient continued to receive therapeutic anticoagulation (which was changed to treatment with rivaroxaban upon discharge because of patient preference). The gradual elevation of his platelet count corresponded to an observed progressive decline in serum-induced activation of platelets in the absence of heparin.

\section{Discussion}

Heparin-induced thrombocytopenia is a wellcharacterized, prothrombotic disorder caused by platelet-activating $\mathrm{IgG}$ antibodies that recognize multimolecular complexes of platelet factor 4 (a cationic protein) bound to anionic heparin through charge-charge interactions. ${ }^{8}$ Platelet activation occurs when the platelet factor 4 /heparin/IgG complexes form in situ on the surface of platelets, cross-linking the platelet Fc $\gamma I I a$ receptors, thereby producing strong platelet activation in association with a marked procoagulant response.
Clinically, heparin-induced thrombocytopenia typically presents 5-10 days after an immunizing exposure to heparin, which is usually heparin given intraoperatively (e.g., during cardiac or vascular surgery) or soon after surgery (e.g., as usual postoperative thromboprophylaxis). ${ }^{12}$ The common occurrence of heparin-induced thrombocytopenia postoperatively is believed to result from the combination of surgery (with the release of platelet factor 4 from activated platelets) and the administration of heparin during or after surgery, triggering the formation of the immunogenic platelet factor $4 /$ heparin complexes. ${ }^{13}$

In our patient, the initial picture of a second decline in the platelet count that began on the fifth postoperative day and while the patient was receiving low-molecular-weight heparin was consistent with heparin-induced thrombocytopenia. However, as the case evolved, several points seemed to argue against the diagnosis, in particular, the progressive decline of the patient's platelet count for nearly a week following the cessation of heparin treatment. One possible explanation for this was concomitant disseminated intravascular coagulation, a well-described complication of heparin-induced thrombocytopenia. ${ }^{8,9}$ However, disseminated intravascular coagulation is unlikely to progress if the patient is receiving effective anticoagulation; our patient was receiving fondaparinux at a therapeutic dose. In addition, stable fibrinogen levels and coagulation parameters (activated partial thromboplastin time and prothrombin time/international normalized ratio) within the normal range suggested that there was no progression to disseminated intravascular coagulation.

It is commonly thought that severe thrombocytopenia (platelet count $<20 \times 10^{9} / \mathrm{L}$ ) argues against a diagnosis of heparin-induced thrombocytopenia; however, it occurs in 5\%-10\% of patients with heparin-induced thrombocytopenia and in about $50 \%$ of patients with delayed-onset heparin-induced thrombocytopenia. ${ }^{9}$ Such patients are at increased risk of disseminated intravascular coagulation and microthrombosis associated with heparin-induced thrombocytopenia. The lowest recorded nadir for a platelet count in a patient with serologically confirmed heparin-induced thrombocytopenia is $2 \times 10^{9} / \mathrm{L} .^{8}$

\section{Delayed-onset heparin-induced thrombocytopenia}

Delayed-onset heparin-induced thrombocytopenia was initially reported in a case series of 12 patients in whom heparin-induced thrombocytopenia began five or more days after their last exposure to heparin. ${ }^{9}$ Recently, the term "delayedonset heparin-induced thrombocytopenia" also 
has been used to describe heparin-induced thrombocytopenia that worsens after heparin treatment ends, ${ }^{8}$ which occurred in our case. Our patient's platelet count recovered relatively slowly; this is consistent with the finding that the normalization of the platelet count may follow a protracted course in patients with delayed-onset heparininduced thrombocytopenia and associated strong reactivity in the absence of heparin in the serotonin release assay. ${ }^{9}$ An evaluation on day 44 after surgery showed that the serum of our patient remained strongly positive in the serotonin release assay at therapeutic heparin doses (Appendix 1). In addition, whereas the reactivity of the serotonin release assay in the absence of heparin declined over time (with a corresponding gradual increase in the patient's platelet count to $61 \times 10^{9} / \mathrm{L}$ ), there was still some residual reactivity $(10 \%$ serotonin release), which explains the persisting degree of thrombocytopenia in the patient. At day 88 after surgery, the platelet count was $111 \times 10^{\circ} / \mathrm{L}$, and there was essentially no reactivity in the serotonin release assay in the absence of heparin (3\% serotonin release).

\section{Diagnostic testing for heparin-induced thrombocytopenia}

The need to make a timely diagnosis and start appropriate therapy requires an awareness of how heparin-induced thrombocytopenia can present and progress in a patient, because it often takes several days to confirm the diagnosis with laboratory tests. However, these tests are critical and can inform the clinician's understanding and characterization of the disease process.

Enzyme-linked immunosorbent assay is undertaken initially and identifies the presence of high levels of antiplatelet factor 4/heparin antibodies in patient serum; its high sensitivity (about $99 \%)^{14,15}$ allows heparin-induced thrombocytopenia to be ruled out if the result is negative, whereas the strength of a positive result can predict the likelihood of true heparin-induced thrombocytopenia. As in our patient, higher optical density levels (>2.00) have been strongly associated with the presence of heparin-dependent, platelet-activating IgG antibodies, which are implicated in heparin-induced thrombocytopenia: up to $90 \%$ of patients with optical density levels greater than 2.0 in the ELISA will have a positive result in the serotonin release assay. ${ }^{16,17}$ When the optical density is not considered, the specificity of a positive ELISA result is limited and has been estimated to be as low as $26 \%$. $^{18}$

As described earlier, the presence of antiplatelet factor 4/heparin antibodies in a patient's serum is not sufficient for a diagnosis of heparin-induced thrombocytopenia. In fact, the serum of most patients with such antibodies will not be able to activate platelets in a serotonin release assay, which indicates that these antibodies are not pathogenic. Conversely, in most true cases of heparin-induced thrombocytopenia, the serotonin release assay will demonstrate the ability of the patient's serum to induce platelet activation only at therapeutic concentrations of heparin, with minor or no platelet activation in the absence of heparin, and an inhibition of platelet activation at very high concentrations of heparin. ${ }^{9}$ Currently, the serotonin release assay is performed in only one laboratory in Canada (McMaster Platelet Immunology Laboratory, Hamilton, Ont.).

The serotonin release assay provides three important contributions to our case. First, the test is more specific for heparin-induced thrombocytopenia than the ELISA and therefore strongly suggests a diagnosis of heparin-induced thrombocytopenia, despite our patient's atypical clinical features. Second, the strong, serum-induced platelet activation at a concentration of $0 \mathrm{IU} / \mathrm{mL}$ heparin is characteristic of delayed-onset heparin-induced thrombocytopenia, which helps to explain both the dramatic progression of thrombocytopenia in the absence of further treatment with heparin and the subsequent slow recovery of the platelet count in our patient. The sera of patients with delayed-onset heparininduced thrombocytopenia, when compared with the sera of patients with usual heparin-induced thrombocytopenia, have strong serotonin release ( $\geq 50 \%$, and often $>80 \%$ ) both in the presence and in the absence of therapeutic concentrations of heparin. Serotonin release will be inhibited at a dose of $100 \mathrm{IU} / \mathrm{mL}$ heparin in all sera of patients with heparin-induced thrombocytopenia. ${ }^{9}$ Finally, the serotonin release assay ruled out fondaparinux cross-reactivity as the cause of the progressive decline in the platelet count in our patient while he received this drug.

\section{References}

1. Greinacher A Warkentin TE. Acquired non-immune thrombocytopenia. In: Marder VJ, Aird, WC, Bennett JS, et al., editors. Hemostasis and thrombosis: basic principles and clinical practice. 6th ed. Philadelphia (PA): Lippincott Williams \& Wilkins; 2013:796-804.

2. Taaning E, Svejgaard A. Post-transfusion purpura: a survey of 12 Danish cases with special reference to immunoglobulin $G$ subclasses of the platelet antibodies. Transfus Med 1994;4:1-8.

3. Naqvi TA, Baumann MA, Chang JC. Post-operative thrombotic thrombocytopenic purpura: a review. Int J Clin Pract 2004;58: 169-72.

4. Warkentin TE. How I diagnose and manage HIT. Hematology Am Soc Hematol Educ Program. 2011;2011:143-9.

5. Lee GM, Arepally GM. Heparin-induced thrombocytopenia Hematology Am Soc Hematol Educ Program. 2013;2013:668-74.

6. Linkins LA, Dans AL, Moores LK, et al. Treatment and prevention of heparin-induced thrombocytopenia: antithrombotic therapy and prevention of thrombosis, 9th ed: American College of Chest Physicians Evidence-Based Clinical Practice Guidelines. Chest. 2012; 141(2 Suppl): e495S-530S.

7. Warkentin TE. Anticoagulant failure in coagulopathic patients: 
PTT confounding and other pitfalls. Expert Opin Drug Saf 2014; 13:25-43.

8. Warkentin TE. Agents for the treatment of heparin-induced thrombocytopenia. Hematol Oncol Clin North Am 2010;24: 755-75.

9. Warkentin TE, Kelton JG. Delayed-onset heparin-induced thrombocytopenia and thrombosis. Ann Intern Med 2001;135:502-6.

10. Warkentin TE, Pai M, Sheppard JI, et al. Fondaparinux treatment of acute heparin-induced thrombocytopenia confirmed by the serotonin-release assay: a 30-month, 16-patient case series. J Thromb Haemost 2011;9:2389-96.

11. Warkentin TE. Fondaparinux: Does it cause HIT? Can it treat HIT? Expert Rev Hematol 2010;3:567-81.

12. Warkentin TE, Kelton JG. A 14-year study of heparin-induced thrombocytopenia. Am J Med 1996;101:502-7.

13. Warkentin TE. HIT paradigms and paradoxes. J Thromb Haemost 2011;9(Suppl 1):105-17.

14. Pouplard C, Amiral J, Borg JY, et al. Decision analysis for use of platelet aggregation test, carbon 14-serotonin release assay, and heparin-platelet factor 4 enzyme-linked immunosorbent assay for diagnosis of heparin-induced thrombocytopenia. Am J Clin Pathol 1999;111:700-6.

15. Bakchoul T, Giptner A, Najaoui A, et al. Prospective evaluation of PF4/heparin immunoassays for the diagnosis of heparininduced thrombocytopenia. J Thromb Haemost 2009;7:1260-5.

16. Warkentin TE, Sheppard JI, Moore JC, et al. Quantitative interpretation of optical density measurements using PF4-dependent enzyme-immunoassays. J Thromb Haemost 2008;6:1304-12.

17. Pearson MA, Nadeau C, Blais N. Correlation of ELISA optical density with clinical diagnosis of heparin-induced thrombocy- topenia: a retrospective study of 104 patients with positive antiPF4/heparin ELISA. Clin Appl Thromb Hemost 2013 Nov. 27

18. Cuker A, Cines DB. How I treat heparin-induced thrombocytopenia. Blood 2012;119:2209-18.

Affiliations: Department of Medicine (Kopolovic), University of Toronto, Toronto, Ont.; Pathology and Molecular Medicine (Warkentin), Hamilton Regional Laboratory Medicine Program, McMaster University, Hamilton, Ont.

Contributors: Ilana Kopolovic drafted the manuscript, and Theodore Warkentin reviewed its intellectual content. Both of the authors approved the final version of the manuscript submitted for publication.

CMAJ invites submissions to "What is your call?" Clinical details (including images) are presented with a multiple-choice question about the diagnosis. The answer and a brief discussion of the condition follow. We specifically invite submissions illustrating common or important radiographic and electrocardiographic diagnoses of appeal to a general audience. We require authors to obtain consent from the patient for publication of his or her story (form available at www.cmaj.ca/site/authors/checklist.xhtml). Submit manuscripts online at http://mc.manuscriptcentral .com/cmaj.

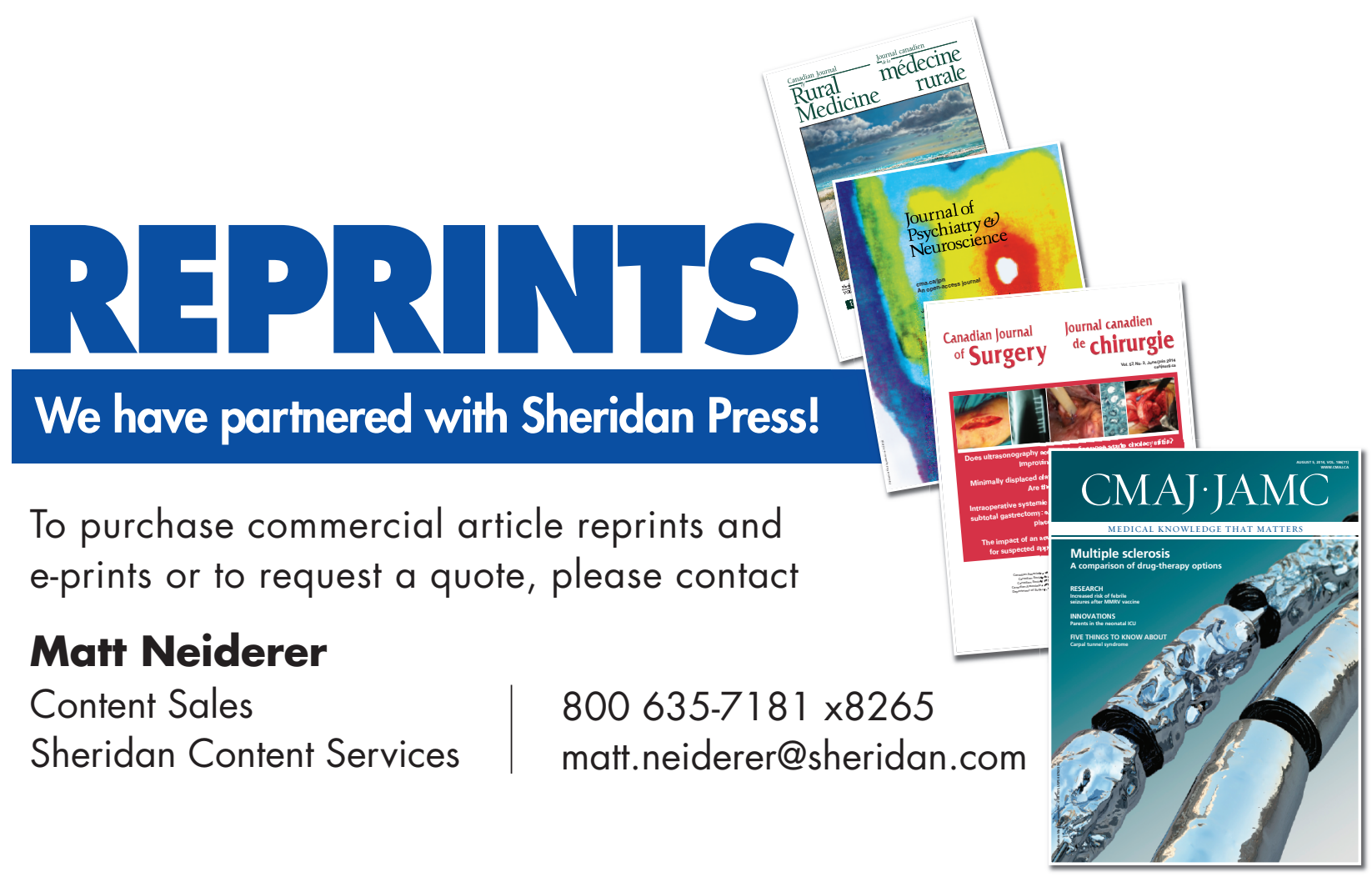

